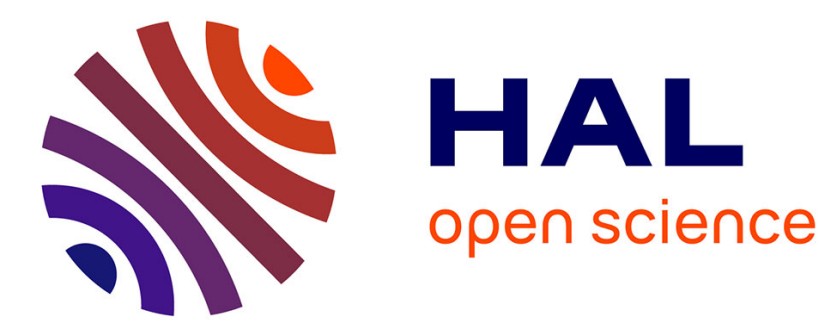

\title{
An Overlooked Consequence of Dietary Mixing: A Varied Diet Reduces Interindividual Variance in Fitness
}

Alistair M. Senior, Shinichi Nakagawa, Mathieu Lihoreau, Stephen J. Simpson, David Raubenheimer

\section{- To cite this version:}

Alistair M. Senior, Shinichi Nakagawa, Mathieu Lihoreau, Stephen J. Simpson, David Raubenheimer. An Overlooked Consequence of Dietary Mixing: A Varied Diet Reduces Interindividual Variance in Fitness. American Naturalist, 2015, 186 (5), pp.649-659. 10.1086/683182 . hal-02105116

\author{
HAL Id: hal-02105116 \\ https://hal.science/hal-02105116
}

Submitted on 20 Apr 2019

HAL is a multi-disciplinary open access archive for the deposit and dissemination of scientific research documents, whether they are published or not. The documents may come from teaching and research institutions in France or abroad, or from public or private research centers.
L'archive ouverte pluridisciplinaire HAL, est destinée au dépôt et à la diffusion de documents scientifiques de niveau recherche, publiés ou non, émanant des établissements d'enseignement et de recherche français ou étrangers, des laboratoires publics ou privés. 


\section{An Overlooked Consequence of Dietary Mixing: A Varied Diet Reduces Interindividual Variance in Fitness}

ARTICLE in THE AMERICAN NATURALIST · SEPTEMBER 2015

Impact Factor: $3.83 \cdot$ DOI: 10.1086/683182

CITATION

5 AUTHORS, INCLUDING:

Shinichi Nakagawa

University of New South Wales

164 PUBLICATIONS 6,058 CITATIONS

SEE PROFILE

French National Centre for Scientific Resea...

33 PUBLICATIONS 434 CITATIONS

SEE PROFILE

David Raubenheimer

University of Sydney

267 PUBLICATIONS 7,777 CITATIONS

SEE PROFILE 


\section{CHICAGO JOURNALS}

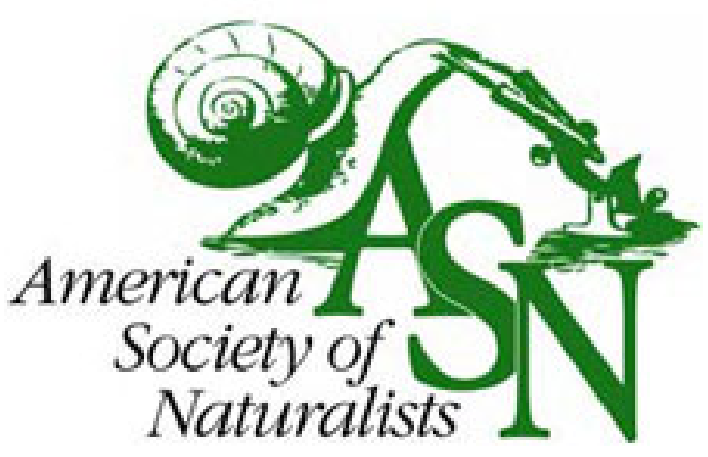

The University of Chicago

An Overlooked Consequence of Dietary Mixing: A Varied Diet Reduces Interindividual Variance in Fitness

Author(s): Alistair M. Senior, Shinichi Nakagawa, Mathieu Lihoreau, Stephen J. Simpson and David Raubenheimer,

Source: The American Naturalist, (-Not available-), p. 000

Published by: The University of Chicago Press for The American Society of Naturalists

Stable URL: http://www.jstor.org/stable/10.1086/683182

Accessed: 02/10/2015 23:22

Your use of the JSTOR archive indicates your acceptance of the Terms \& Conditions of Use, available at http://www.jstor.org/page/info/about/policies/terms.jsp

JSTOR is a not-for-profit service that helps scholars, researchers, and students discover, use, and build upon a wide range of content in a trusted digital archive. We use information technology and tools to increase productivity and facilitate new forms of scholarship. For more information about JSTOR, please contact support@jstor.org. 


\title{
An Overlooked Consequence of Dietary Mixing: A Varied Diet Reduces Interindividual Variance in Fitness
}

\author{
Alistair M. Senior, ${ }^{1, \star}$ Shinichi Nakagawa, ${ }^{2}$ Mathieu Lihoreau, ${ }^{3}$ Stephen J. Simpson, ${ }^{1}$ \\ and David Raubenheimer ${ }^{1,4}$
}

1. Charles Perkins Centre and School of Biological Sciences, University of Sydney, Sydney, New South Wales, Australia; 2. Evolution and Ecology Research Centre, School of Biological, Earth and Environmental Sciences, University of New South Wales, New South Wales, Australia, and Department of Zoology, University of Otago, Dunedin, Otago, New Zealand; 3. Centre National de la Recherche Scientifique and Université Paul Sabatier, Centre de Recherches sur la Cognition Animale, Toulouse, France; 4. Faculty of Veterinary Science, University of Sydney, Sydney, New South Wales, Australia

Submitted April 9, 2015; Accepted June 10, 2015; Electronically published September 28, 2015

Online enhancement: appendix. Dryad data: http://dx.doi.org/10.5061/dryad.9hc76.

ABSTRACT: The determinants of diet breadth are of interest to nutritionists, ecologists, and evolutionary biologists. A recent synthesis addressing this issue found conflicting evidence for the relationship between diet breadth and mean individual fitness. Specifically, it found that while, on average, a mixed diet does increase mean fitness, in some instances, a single food provides equal (or higher) fitness than a mixed diet. Critical to ecological and evolutionary considerations of diet, however, is not only mean fitness but also variance in fitness. We combine contemporary meta-analytic methods with models of nutritional geometry to evaluate how diet affects between-individual variance in fitness within generalist consumers from a range of trophic levels. As predicted by nutritional geometry, we found that between-individual variance in fitness-related traits is higher on single-food than mixed diets. The effect was strong for longevity traits $(57 \%$ higher) and reproductive traits (37\%) and present but weaker for size-related traits (10\%). Further, the effect became stronger as the number of available foods increased. The availability of multiple foods likely allows individuals with differing nutritional optima to customize intake, each maximizing their own fitness. Importantly, these findings may suggest that selection on traits correlated with nutritional requirements is weak in heterogeneous nutritional environments.

Keywords: dietary generalism/specialism, life history, lnCVR, metaanalysis, nutritional ecology, nutritional geometry.

\section{Introduction}

The evolution of niche breadth is a primary question in nutritional ecology, community ecology, and evolutionary biology (Dennis et al. 2011; Kassen 2002; Simpson and Raubenheimer 2012). The determinants of diet breadth are of particular ecological relevance as diet mediates numerous in-

\footnotetext{
* Corresponding author; e-mail: alistair.senior@sydney.edu.au.
}

Am. Nat. 2015. Vol. 186, pp. 000-000. (C) 2015 by The University of Chicago. 0003-0147/2015/18605-56196\$15.00. All rights reserved.

DOI: $10.1086 / 683182$ terspecific interactions, including those between competitors, plants and herbivores, prey and predators, and hosts and their microbiome (Simpson et al. 2015). A widely invoked explanation for the benefits of consuming multiple prey/plant species is the balanced diet hypothesis, which posits that by mixing foods consumers have a more nutritionally balanced diet than could be gained from a single food alone, which in turn translates to higher fitness (DeMott 1998). A recent synthesis collated the results of $161 \mathrm{ex}-$ perimental studies comparing the mean fitness of generalist consumers fed on mixed and single-food diets in the most comprehensive analysis of diet breadth and fitness to date (Lefcheck et al. 2013). Lefcheck et al.'s (2013) analyses found contrasting and heterogeneous evidence for the balanced diet hypothesis; that is, they found that while on average a mixed diet does increase mean fitness, it is possible for single foods to provide equal mean fitness.

Although there is mixed evidence regarding the effects of diet mixing on mean fitness, it remains completely untested as to how a mixed diet affects between-individual variance in fitness. Despite being largely overlooked in this context, between-individual variation underpins selection and drives adaptation (Hallgrimsson and Brian 2005; Orr 2009). Furthermore, at the community level, ecologists have recently advocated the benefits of more variance-focused approaches in understanding how niche breadth allows multiple species to coexist and how communities are structured (Violle et al. 2012). Finally, experimental and ecological data have demonstrated important interactions between within-population variance and niche breadth (Kassen 2002; Van Valen 1965).

Considering diet, within a group, population, or species, there is likely to be considerable interindividual variability in nutritional requirements (Lihoreau et al. 2015). For example, in many species, the requirements and foraging pri- 
orities of the sexes differ. A study on captive field crickets (Teleogryllus commodus) exemplifies such variability, demonstrating that males maximize their reproductive performance on a high-carbohydrate diet, whereas females perform better on a more protein-rich diet (Maklakov et al. 2008). Many animals also live in groups or populations that simultaneously contain individuals of differing developmental stages, and there is abundant evidence that nutritional requirements change throughout an individual's life cycle (Raubenheimer et al. 2007; Simpson and Raubenheimer 2012). Social insects such as ants, for example, live in colonies with overlapping generations, where optimal diets range from the protein-rich diet needed by growing larvae to the carbohydrate-rich diet eaten by nonreproductive adults for colony maintenance (Dussutour and Simpson 2009). Finally, even where individuals appear the same sex and age, heterogeneity in many other traits that may correlate with nutritional requirements is readily observable; examples include between-individual variation in metabolic rate, body size, and life-history strategy (Cam et al. 2002; Honěk 1993; Huchard et al. 2014; Lim et al. 2014; Mathot and Dingemanse 2015). Given the probable ubiquity of intraspecific variability in nutritional requirements, how may a mixed diet affect interindividual variance in fitness?

A powerful conceptual tool with which to consider this fundamental question is nutritional geometry (summarized in fig. $1 A, 1 B$ ), a state-space modeling approach for studying how animals meet their requirements for multiple nutrients (Raubenheimer and Simpson 1993; Simpson and Raubenheimer 2012; Simpson et al. 2015). At their most simplistic, nutritional geometry models depict two nutrients in a Cartesian coordinate system known as the nutrient space (fig. 1A). Central to this approach is the concept that a coordinate (or broader region) within the nutrient space, known as the intake target (IT), denotes an animal's nutritional requirements (i.e., ideal amount and balance of nutrients; fig. 1A). Animals attempt to reach this coordinate by eating the foods available in their environment (fig. $1 B$ ). While a number of ITs may be quantifiable for different traits (e.g., to maximize growth rate or longevity) and developmental stages (e.g., juvenile or adult), evolutionary theory suggests that, when possible, animals should attempt to reach an IT that maximizes evolutionary fitness, a prediction that is supported by experimental data (Jensen et al. 2012; Simpson and Raubenheimer 2012; Simpson et al. 2004).

In geometric models, interindividual variability in nutritional requirements can be visualized by a distribution of ITs, each maximizing the fitness of an individual, surrounding the group, population, or species mean (fig. $1 C$; Lihoreau et al. 2015; Senior et al. 2015a). Using this approach, novel predictions can be generated for how diet mixing affects between-individual variance in fitness. As shown in figure $1 D$, a diet made up of more than one food can allow each individual to reach its own IT. The consequence of mixing foods may thus be that any between-individual variance in fitness attributable to individual dietary requirements is low. In contrast, being confined to a single food rail is likely to result in some individuals getting closer to their IT than others, inducing variation in fitness (fig. $1 D$ ).

We investigated the effects of dietary mixing on betweenindividual variance in fitness within generalist species, using a recently defined metric for meta-analysis of variance, the $\log$ coefficient variance ratio (lnCVR; Nakagawa et al. 2015). Based on nutritional geometry, we hypothesize that a mixed diet reduces interindividual variance in fitness, while increasing mean fitness (fig. 1D).

\section{Material and Methods \\ Data Extraction}

To maintain a high standard of reporting, we follow the preferred reporting items for systematic review and metaanalyses (PRISMA) protocol (Liberati et al. 2009). The protocol was developed for review of clinical studies, and it has been suggested that many (although not all) PRISMA apply to ecological and evolutionary meta-analyses (Nakagawa and Poulin 2012).

We started with the 161 articles from which Lefcheck et al. (2013) extracted their data. To these articles, we applied our five inclusion criteria: (1) The study must state a quantitative measure of mean and interindividual variance of a fitnessrelated trait and an associated sample size (or measures from which these data were attainable; e.g., degrees of freedom, df). These data are required to estimate effect sizes and measurement errors. Of the 161 articles, 85 failed to meet this criterion. (2) Treatment groups must have been independently fed at least one single-food diet and one diet of $>1$ food incorporating the same food as that on which the single-food group was fed; for example, if the food comprising the single-food diet was not included in any mixed diet in the study, then those data were deemed incomparable. We define a single-food diet as a diet composed of one artificial food or plant/prey species belonging to the same genera (although this was usually one species). One article failed to meet this criterion. (3) Fitness quantifications must come from $>2$ consumers, as is necessary for accurate estimation of effect sizes. Two articles failed to meet this criterion. (4) The experiment must allow individuals to mix their diet in response to their nutritional requirements. Treatments where animals were fed different foods sequentially were not included. Two articles failed to meet this criterion. (5) The study must report fitness-related traits for animals. Studies on plants were excluded to minimize the potentially confounding effects of vast phylogenetic differences. One article failed to meet this criterion. 

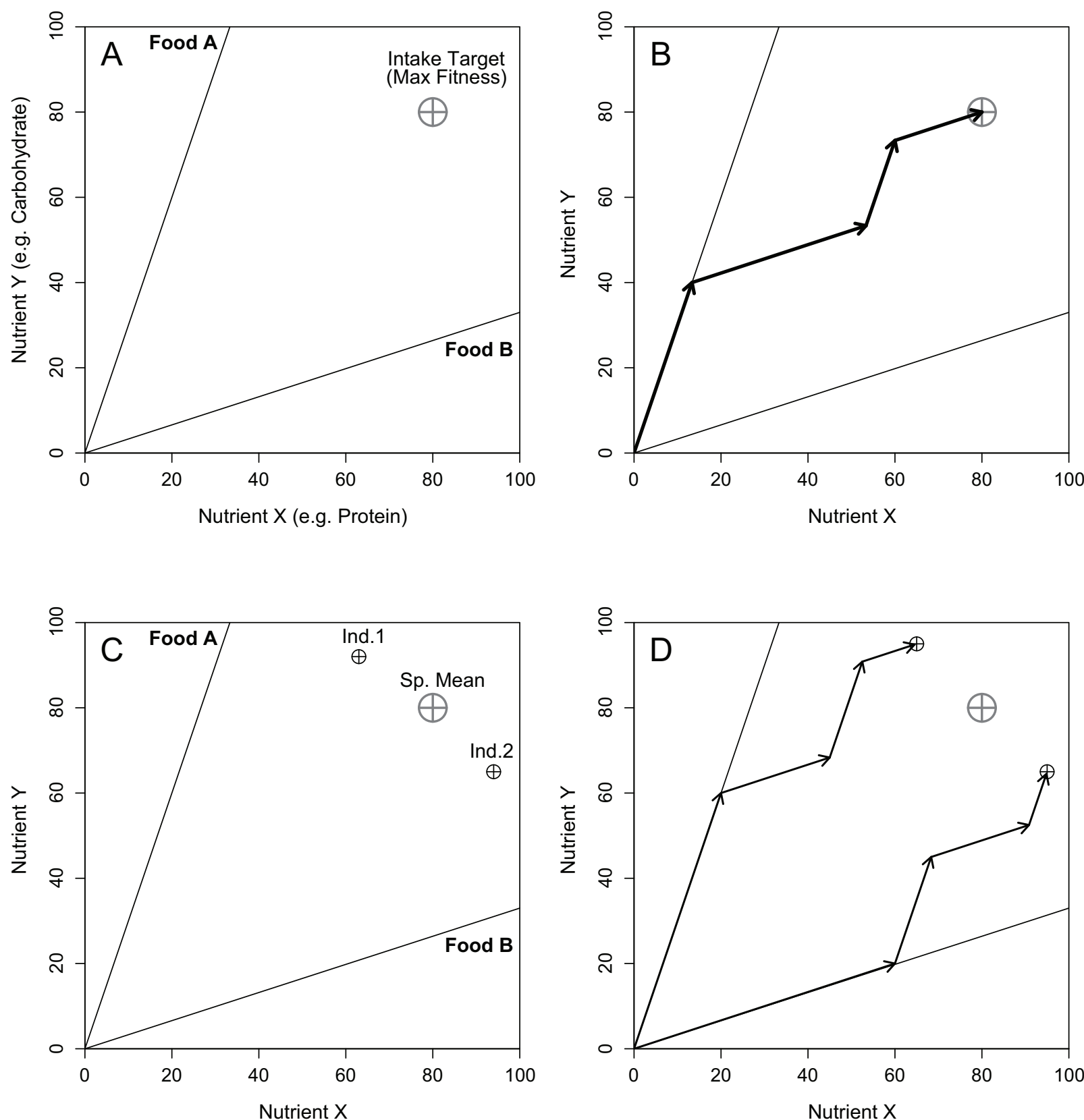

Figure 1: $A$, Nutritional geometry depicting the nutrient space for two nutrients: nutrient $\mathrm{X}$ (e.g., protein; $X$-axis) and nutrient $\mathrm{Y}$ (e.g., carbohydrate; $Y$-axis). Radials (food rails) depict nutrient ratios in foods (e.g., food A rich in nutrient $Y$ and food B rich in nutrient $\mathrm{X}$ ). The intake target (IT) represents the amount and balance of the nutrients that maximize fitness. $B$, While eating, an individual's nutritional state (coordinate) moves through the space in parallel with the rail for the food being eaten. Here, the individual reaches the IT by mixing the two foods. $C$, Interindividual differences in nutritional requirements are represented by individual (Ind.) ITs surrounding the species (Sp.) mean. $D$, To reach their ITs, individual 1 eats mostly food A and individual 2 eats mostly food B. Because both individuals reach their IT, variance in fitness may be low. If confined to one food, variance in fitness might be high; for example, if only food A were available, individual 1 may have higher fitness than individual 2.

Seventy articles met our criteria, although one had been retracted from publication, leaving 69 (see fig. A1 and "Data Bibliography" in the appendix for a PRISMA-style flow diagram and references; appendix and figs. A1-A4 are available online). Many articles failed to meet the first criteria because they reported measures of population fitness (e.g., zooplankton population growth rates) or reported fitness estimated from several replicates, with each comprising multiple indi- 
viduals, and averaged away the between-individual (withinreplicate) variance we are interested in. From the remaining articles, estimates of mean and within-group variance on the following three fitness-related traits (those used by Lefcheck et al. 2013) were extracted from each treatment group: (1) size-related traits, (2) reproduction-related traits, and (3) longevity-related traits. Growth rate was the preferred size measure (this appeared the most widely reported), although data on mass and structural size were used where growth was unavailable. For reproduction, we prioritized total reproductive output, although other traits such as gonadal indexes were included. All longevity estimates were for life span. Where possible, we extracted all available trait data, although most articles (64/69) reported data for only one trait and the remaining five articles for only two traits; note that differences between traits were explored with metaregression (described below). Data from diets that were obviously inadequate (e.g., all consumers died within the first few days or had a negative growth) were excluded. Where fitness measures were quantified over time, we extracted data from the last measurement in the trial for which comparable data were available. In some instances, we made assumptions about sample size; for example, where data were based on all survivors, the starting sample size was adjusted by survival. We also extracted moderator data (described below). Any relevant data, such as estimates of mean and variance in fitness traits, reported in graphical form were extracted using the software GraphClick (Boyle et al. 2013).

Our library is unlikely to constitute all studies comparing the fitness of groups of animals fed mixed and singlefood diets. However, meta-analyses do not necessarily need to be comprehensive as long as the process for gathering articles is not biased (Jennions et al. 2012), and there is no reason to believe the library collected by Lefcheck et al. (2013) would be biased with regard to our hypotheses.

\section{Effect Sizes}

We used two effect sizes to compare fitness on mixed and single-food diets. To compare mean fitness, we used the $\log$ response ratio (hereafter, $\operatorname{lnRR}$ ) and its measurement error variance $\left(s_{\operatorname{lnRR}}^{2}\right)$, calculated following equations (5) and (6) in Nakagawa et al. (2015); this analysis is analogous to that by Lefcheck et al. (2013) but applied to the subset of their library that is usable with $\operatorname{lnCVR}$. To compare variance in fitness between the two dietary groups, we calculated $\operatorname{lnCVR}$ and its measurement error variance $\left(s_{\operatorname{lnCVR}}^{2}\right)$ following equations (11) and (12) in Nakagawa et al. (2015). Importantly, lnCVR allows one to make formal meta-analytic comparisons between the variance of groups after correcting for differences in variance that may be expected given changes in the mean (Nakagawa et al. 2015). Effect sizes were calculated as a positive/negative mean effect constituting higher/lower mean (lnRR) or coefficient of variance (lnCVR) of fitness in the group fed a single food. To aid interpretation, in the results, we exponentiated meta-analytic mean effects to get raw ratios between groups and associated percentage differences.

We calculated all possible effects within an experiment; for example, if the study contained two single-food and two mixed diets, there are four possible comparisons and we calculated four effects. This method does, however, lead to correlated structures (i.e., covariance), sometimes referred to as stochastic dependency, where effect sizes are based on the same treatment data (Gleser and Olkin 2009). Lefcheck et al. (2013) dealt with such nonindependence by averaging variation in fitness on different single-food and mixed diets. We did not use this approach for three reasons: (1) averaging variation leads to a loss of power; (2) for meta-analysis, we require estimates of sampling error for which data would have to be approximated for averaged values; and (3) averaged estimates may not necessarily represent the original data, meaning that in the final analysis, mean estimates may be unrepresentative and statistical heterogeneity underestimated. Covariance between effect sizes that share data can be estimated (Gleser and Olkin 2009; Lajeunesse 2011). For $\operatorname{lnRR}$, covariances were estimated following Lajeunesse (2011). For lnCVR, covariances were estimated as outlined in the appendix ("Covariance of $\operatorname{lnRR}$ and $\operatorname{lnCVR}$ "). The analyses in the main text make partial corrections using estimated covariances. In the appendix ("Random Slopes MetaRegression Approach"), we also report alternative analyses that are free from issues of stochastic dependency and explore the sensitivity of our results to other potential shortcomings of the lnCVR metric (discussed in Nakagawa et al. 2015). These analyses produced qualitatively similar results to those described in the main text ("Covariance of $\operatorname{lnRR}$ and lnCVR" in the appendix). Analyses were performed in $\mathrm{R}$, version 3.1.1 (R Development Core Team 2014).

\section{Meta-Analytic Procedures}

We first fitted random-effects meta-analyses (REMA) using the rma function in the R package metafor (Viechtbauer 2010). Two models were implemented, one each with $\ln R R$ and $\ln C V R$ as the response, along with associated measurement errors $\left(s_{\operatorname{lnRR}}^{2}\right.$ and $\left.s_{\operatorname{lnCVR}}^{2}\right)$. Meta-analysis, like most statistical approaches, assumes that data are independent, yet effect sizes may covary when they come from the same study or model organism or even when they use data from the same treatment groups. Such issues of nonindependence can be modeled using multilevel meta-analyses (MLMAs) with random factors (i.e., linear mixed models; Nakagawa and Santos 2012). We implemented MLMAs as linear mixed models (Nakagawa and Santos 2012) using the MCMCglmm function in the package MCMCglmm (Hadfield 2010). One 
MLMA was specified for each effect size along with its estimated measurement error, random factors giving identities for the article of origin and the experimental species, and a matrix giving the estimated covariance between effect sizes that make comparisons to the same mixed-diet data. In analyses of different species, the degree of independence between effects may also be affected by the evolutionary ancestry that species share (Lajeunesse et al. 2013). We explored phylogenetic effects by incorporating phylogenetic covariances (Hadfield and Nakagawa 2010). However, these analyses were sensitive to specifications of the phylogeny, and in no instances did including the phylogeny improve model fit. We also found no evidence for phylogenetic effects; thus, phylogenetic models are left out of the main text (details of phylogenetic analyses and other MCMCglmm specifications are outlined in appendix sections "Phylogenetic MetaAnalyses" and "Parameters for MCMCglmm and Model Convergence"; table A2; figs. A2, A3; tables A1-A5 are available online). Statistical significance of a mean estimate (est.) was inferred when the $95 \%$ confidence/credible interval (CI) did not cross zero.

An underlying assumption in meta-analysis is that variation between the observed effects can be explained by the fact that some effects are more precisely estimated than others (i.e., the measurement errors included in our model). However, there may also be true differences between the observed effects, where, for example, data come from different species. This, "true" variance is referred to as heterogeneity in a meta-analytic context and can be estimated (Higgins and Thompson 2002). To estimate heterogeneity, we present total $I^{2}$ (the percentage of variance in effects that cannot be explained by measurement error) and $I^{2}$ partitioned among the random factors fitted to MLMAs, following Nakagawa and Santos (2012). Throughout, we refer to meta-analytic heterogeneity as $I^{2}$ to avoid confusion with biological heterogeneity. We interpreted $I^{2}$ of $25 \%, 50 \%$, and $75 \%$ as small, medium, and large, respectively (Higgins and Thompson 2002). Based on Lefcheck et al. (2013), it seems likely that there will be high $I^{2}$ in at least $\operatorname{lnRR}$. To explore $I^{2}$, we fitted moderators as fixed factors in linear mixed models, that is, multilevel meta-regression (MLMR; using the MLMA framework described above). Moderators were: (1) Trait.Typethe type of fitness trait measured as a three-level predictor (longevity, reproduction, or size); (2) Habitat - the habitat of the organism fitted as a two-level predictor (terrestrial or marine); (3) Trophic.Level - a two-level predictor describing the trophic level of the experimental species (primary or secondary; where experimental diets were exclusively plant based, the consumer was defined as primary, while all others, including omnivorous diets, were secondary); (4) Defensea two-level predictor describing whether the food source had a toxin defense (yes or no); and (5) Breadth - the number of foods comprising the mixed $\operatorname{diet}(Z$ transformed to aid in- terpretation; Schielzeth 2010). Where possible, we also explored the influence of experimental duration but found no significant effect (see "Effects of Experimental Duration" in the appendix). Predictors were explored using deviance information criterion (DIC; Spiegelhalter et al. 2002), and we restricted ourselves to additive models because of the unbalanced nature of our data set.

We explored publication bias (systematic bias in the available data, owing to the fact that significant results are more likely to be published) by looking for asymmetry in funnel plots. This method alone can be misleading, especially where one expects high heterogeneity. Thus, a modified version of Egger's regression and funnel plots were also applied to meta-analytic residuals from MLMAs, which may be less sensitive to funnel plot asymmetry resulting from heterogeneity (Egger et al. 1997; Nakagawa and Santos 2012). Where detected, the potential effects of publication bias on our metaanalytic estimates were explored using trim and fill analysis, implemented using the trimfill function in the R package metafor (Viechtbauer 2010).

\section{Results}

We obtained 392 estimates of mean and variance in fitnessrelated traits on single-food diets (323 size related, 47 reproduction related, and 14 longevity related) and 249 estimates from mixed diets (198 size, 35 reproduction, and 16 longevity). These data translated to 818 estimates of each lnRR and lnCVR: 642 effects for size, 120 for reproduction, and 56 for longevity. These data came from 71 species (appendix, fig. A2) representing 13 classes, 28 orders, and 46 families. By species, Insecta was the most well-represented class (18 species). Forty-two organisms were classified as primary consumers and 29 as secondary (on the basis of experimental diets); 45 were marine and 26 terrestrial. All data and code are available from the Dryad Digital Repository: http://dx .doi.org/10.5061/dryad.9hc76 (Senior et al. 2015b). ${ }^{1}$

REMA of $\operatorname{lnRR}$ estimated a negative statistically significant effect (REMA est., CI for $\operatorname{lnRR}=-0.363,-0.408$ to -0.318 ; fig. 2), which exponentiates to a ratio of mean of fitness-related traits on single-food diets to mixed diets of 0.700 ; that is, mean fitness is $30 \%$ lower on single foods. REMA of $\operatorname{lnCVR}$ estimated a statistically significant positive estimate (REMA est., CI for $\operatorname{lnCVR}=0.217,0.181$ to 0.254 ; fig. 2), which transformed to an average ratio of coefficient of variation of fitness-related traits on single-food to mixed diets of 1.242; that is, variance in fitness is $24 \%$ higher on single-food diets than mixed diets. The MLMA analysis, which made corrections for correlated structures es-

1. Code that appears in The American Naturalist is provided as a convenience to the readers. It has not necessarily been tested as part of the peer review. 


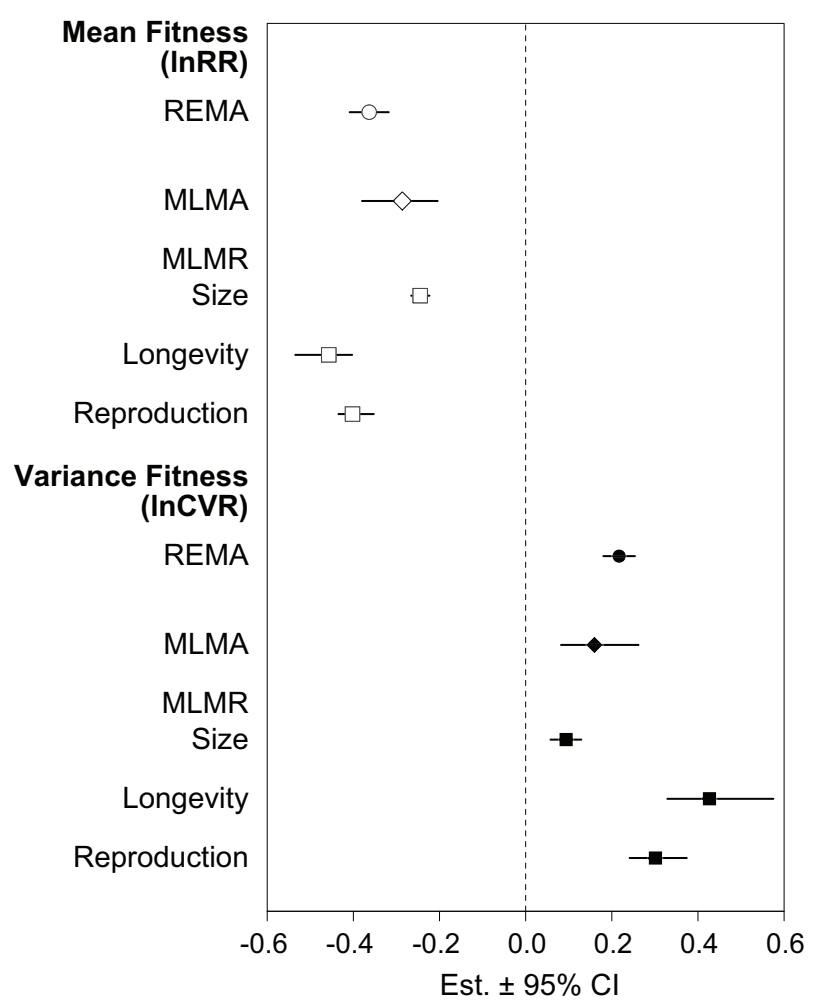

Figure 2: Meta-analytic estimates (Est.) and 95\% confidence/credible intervals (CIs) from random-effects meta-analyses (REMA) and multilevel meta-analyses (MLMAs) and multilevel meta-regression (MLMR) of differences in mean ( $(\mathrm{nRR})$ and variance $(\mathrm{lnCVR})$ in fitness of treatment groups fed single-food and mixed diets. Positive/negative estimates suggest higher/lower measures of fitness-related traits among animals fed single-food diets. Estimates with a 95\% CI not crossing zero are statistically significant. MLMA/MLMR estimates are based on the mode of the posterior distribution. MLMR estimates are overall estimates for each fitness-trait-based model-averaged coefficient (table 2).

timated similar effects to REMA (MLMA est., CI for $\operatorname{lnRR}=$ $-0.286,-0.380$ to -0.204 ; MLMA est., CI for $\operatorname{lnCVR}=$ $0.160,0.082$ to 0.262 ; fig. 2 ).

As expected, there was very high total $I^{2}$ in $\ln R R(\sim 99 \%$; table 1$)$, but high $I^{2}$ was also present for $\operatorname{lnCVR}(\sim 80 \%$; table 1), indicating that the effects of diet on fitness-related traits are not always consistent. Small amounts of $I^{2}$ were partitioned into article and species random factors in MLMA of $\operatorname{lnCVR}$, leaving medium levels unexplained (table 1). For $\operatorname{lnRR}$, a small amount of $I^{2}$ was ascribed to the article of origin (table 1). Based on DIC, there was no one clear best model for $\operatorname{lnRR}$ or $\operatorname{lnCVR}$; thus, we used model averaging to attain MLMR estimates (table 2; see appendix section "Model Averaging" and tables A3-A5 for details; for an overview of model averaging, see Grueber et al. 2011).

Trait type consistently featured in the best-fitting models (appendix; tables A3, A4) and strongly affected both mean and variance, with longevity and reproduction show- ing stronger negative effects for $\ln R R$ and stronger positive effects for lnCVR than size-related traits (fig. 2; table 2). MLMR estimates translate to mean size-, longevity-, and reproduction-related traits being $22 \%, 38 \%$, and $32 \%$ lower on single-food than mixed diets, respectively. MLMR estimates for lnCVR translate to the coefficient of variance for size, longevity, and reproduction traits being on average around $10 \%, 57 \%$, and $37 \%$ higher on single-food than mixed diets, respectively. Terrestrial organisms were estimated to have higher $\ln R R$ than aquatic organisms, although the overall difference was rather small, and there was no significant effect of habitat on InCVR (table 2). The trophic level of the consumer and the presence of a toxic defense had noticeable effects, with larger negative $\ln R \mathrm{R}$ and larger positive lnCVR observed in secondary consumers and when toxins were present (table 2). Finally, as the number of foods in the mixed diet increased, effects became more pronounced: an increase in breadth of the mixed diet being associated with decreasing $\ln R \mathrm{R}$ and increasing $\operatorname{lnCVR}$ (table 2).

For $\operatorname{lnRR}$, funnel plots of raw data suggested asymmetry indicative of publication bias, although those of MLMA residuals looked more symmetrical (fig. A4A, A4B). Regression tests of asymmetry detected no evidence for publication bias (intercept est., $\mathrm{CI}=0.403,-0.300$ to 1.106 ). For $\operatorname{lnCVR}$, no asymmetry was obvious in funnel plots (fig. A4C, A4D). However, regression tests of asymmetry produced a statistically significant positive intercept, suggesting bias (intercept est., CI $=0.402,0.150$ to 0.653 ). Trim and fill analyses produced mixed evidence for publication bias in lnCVR. Using the L0 estimator, trim and fill suggested 81 effects were missing from the left of the plot, and inclusion of those effects would adjust the MLMAestimated meta-analytic mean by -0.073 (adjusted MLMA est. $=0.087)$. However, the R0 estimator, which incorporates a null hypothesis test, suggested no statistically significant publication bias ( $\mathrm{R} 0$ missing studies est. $\pm \mathrm{SE}=2$ $\pm 2.450, P=.125)$. We feel that the L0 estimator is overly

Table 1: Heterogeneity $\left(I^{2}\right)$ estimates

\begin{tabular}{lcccc}
\hline Model & Total & Article & Species & Residual \\
\hline lnRR: & & & & \\
REMA & 99.70 & $\ldots$ & $\ldots$ & $\ldots$ \\
MLMA & 99.68 & 9.579 & 1.960 & 88.14 \\
lnCVR: & & & & \\
REMA & 80.80 & $\ldots$ & $\ldots$ & $\ldots$ \\
MLMA & 79.51 & 8.643 & 15.93 & 54.93 \\
\hline
\end{tabular}

Note: Shown are estimates of $I^{2}(\%)$ produced by random-effects meta-analysis (REMA) and multilevel meta-analyses (MLMA) of differences in mean (lnRR) and variance (lnCVR) in fitness of consumers fed single-food and mixed diets. For MLMA analysis, total $I^{2}$ was partitioned into that explained and unexplained (residual) by those random factors fitted to the model (Nakagawa and Santos 2012). For MLMA, $I^{2}$ is based on the mode of the posterior distribution. 
Table 2: Model-averaged coefficients from meta-regression

\begin{tabular}{|c|c|c|c|c|c|c|}
\hline \multirow[b]{2}{*}{ Parameter } & \multicolumn{3}{|c|}{$\ln R R$} & \multicolumn{3}{|c|}{$\operatorname{lnCVR}$} \\
\hline & Est. & LCI & UCI & Est. & LCI & UCI \\
\hline Intercept & -.245 & -.266 & -.224 & .094 & .058 & .129 \\
\hline Trait.Type $e_{\text {longevity }}$ & -.229 & -.290 & -.160 & .357 & .233 & .483 \\
\hline Trait.Type ${ }_{\text {reproduction }}$ & -.141 & -.189 & -.106 & .219 & .152 & .287 \\
\hline Habitat $_{\text {terrestrial }}$ & .024 & .007 & .052 & -.005 & -.044 & .042 \\
\hline Trophic.Level $_{\text {secondary.consumer }}$ & -.077 & -.104 & -.052 & .073 & .032 & .110 \\
\hline Defense $_{\text {yes }}$ & -.037 & -.084 & -.008 & .154 & .068 & .260 \\
\hline Z.Breadth & -.034 & -.047 & -.027 & .024 & .012 & .040 \\
\hline
\end{tabular}

Note: Shown are model-averaged posterior mode estimates (Est.) and lower to upper 95\% credible intervals (LCI/UCI) of moderators fitted to multilevel meta-regressions of the effect sizes for differences in mean fitness (lnRR) and variance in fitness (lnCVR) between groups of consumers fed single-food and mixed diets. Estimates with a LCI to UCI excluding zero may be considered statistically significant.

conservative here and further address the issue of publication bias in the following section.

\section{Discussion}

We found that the mean fitness of consumers on a singlefood diet was lower than that of those on a mixed diet, but between-individual variance in fitness was higher among those on single-food diets. However, both of these effects show a large degree of inconsistency (i.e., total $I^{2}$ ). We were able to attribute a portion of $I^{2}$ to the article of origin in analyses of both $\operatorname{lnRR}$ and $\operatorname{lnCVR}$, and species specificity appeared important only in the case of variance. The attribution of even a low degree of $I^{2}$ to article of origin suggests that the specifics of the experimental diets used affect variation between effect sizes. Meta-regression was further supportive of this, demonstrating that as a diet is made of more foods, the mean of fitness-related traits increases and variance in those traits decreases.

Meta-regression also suggested that ecological factors such as the trophic level of the consumer may be of some importance, but perhaps the most important moderator was the fitness trait under observation. Considering $\operatorname{lnCVR}$ specifically (our main analysis of interest), a single-food diet appears to induce particularly high variance in longevity and reproductive capacity. From this result, we may infer that there is considerable between-individual variation in the intake target that maximizes those traits; certain individuals live longer/have higher reproductive ability than others on singlefood diets. We also observed that variance in size-related traits is increased when diets are confined to a single food, but to a lesser extent than other traits. Variance in size traits may appear less affected than other traits by dietary breadth, because size measurements (particularly mass) are inaccurate predictors of performance. That is to say, individuals on different diets (or with differing nutritional needs but on the same diet) may gain mass at equal rates, though some indi- viduals gain lean mass, whereas others build up higher stores of fat.

Trim and fill analyses of $\operatorname{lnCVR}$ produced mixed evidence of publication bias. It is unclear how the theoretical basis for publication bias can be applied to variance. Reduced between-individual variance within a treatment group would increase the precision of an estimated treatment mean, increasing the likelihood of detecting statistically significant differences between treatment groups (making publication more likely); for a given sample size, variance and standard error are positively correlated. However, reduced variance in either treatment group should equally affect publication. Thus, we cannot envisage how publication bias would systematically affect the reporting of variance in only one group, as suggested by our analyses (i.e., single-diet treatments). Although it has been suggested that publication bias tools can be readily applied to lnCVR (Nakagawa et al. 2015), future theoretical work may be needed to assess the applicability of these methods to the metric.

Both our and Lefcheck et al.'s (2013) analyses show that across the entire data set, a mixed diet increases the mean of fitness-related traits relative to a single-food diet but that this effect is variable, evidenced by high total (and residual) statistical heterogeneity $\left(I^{2}\right)$ in the analysis of $\ln R R$ here and by data subsetting in Lefcheck et al.'s (2013) analyses. Using nutritional geometry, we can identify three dietary mechanisms that may contribute to this inconsistency between effects (summarized in fig. 3). First, depending on the nutritional composition of the foods in the diets relative to the IT, there are certain scenarios in which fitness should not be higher on a mixed diet than on a single-food diet. For example, in comparisons of mixed diets to single-food diets made up of a nutritionally balanced food with a food rail passing directly through the IT, the mixed diet cannot provide higher fitness than the single-food diet (fig. $3 A$ ). Although for this to apply, we note that between-individual variance in nutritional requirements must be low. 

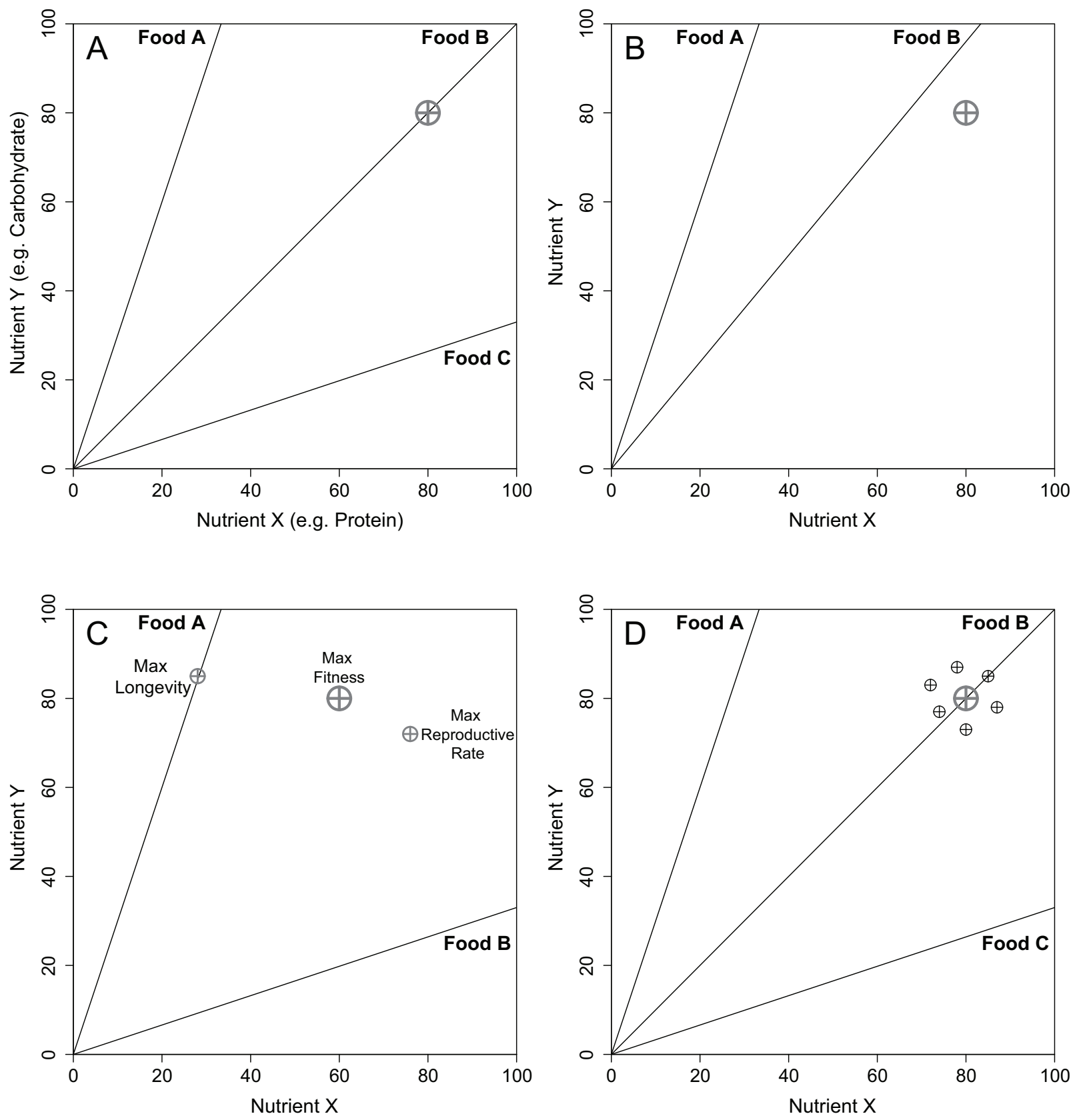

Figure 3: $A$, Here, mixed diets comprising foods in any combination cannot provide higher fitness than a diet solely of food B. $B$, In some environments, sampling available foods may constitute an exploratory cost. Here, sampling food A will cause an animal's nutritional state to move further away from the intake target (IT) than if the animal were experimentally confined to food B. C, Differing ITs may maximize reproductive rate, longevity, and fitness. A single-food diet comprising food A would maximize longevity, but on a mixed diet, individuals eat foods to reach the IT that maximizes fitness. Consequently, longevity is reduced on the mixed diet. $D$, ITs of individuals with differing nutritionally correlated traits are indicated by the small black crosshairs, surrounding the species mean. Food B will meet the average species requirements, but within-population variation can prevent the species from specializing. The removal of food A and/or C may reduce within-population variation in ITs and correlated traits.

Second, when faced with multiple nutritionally imbalanced foods, an animal may sample all available foods in order to try and rectify an imbalanced nutritional state. However, the process of sampling foods may actually exacerbate nutritional imbalance, constituting an exploratory cost to diet mixing. For example, if experimentally fed the diet in figure $3 B$, an animal may sample food A, which would have the ultimate effect of pulling the animal's nutritional state 
further away from the IT than if it were experimentally confined to food B. Indeed, theoretical and empirical evidence support the idea that animals should display exploratory feeding behaviors, or some level of impulsivity, regularly sampling available foods, especially when faced with nutritionally imbalanced dietary options (Day et al. 1998; Houston et al. 2011).

Third, here we do not have measures of fitness but rather a suite of fitness-related traits that organisms may trade off against one another. Thus, it may be possible for single-food diets to optimize one trait, but when offered a mixed diet, organisms may navigate to another IT that maximizes fitness by trading off fitness-related traits (fig. $3 C$ ). A clear example of this consequence of diet mixing was demonstrated by Lee et al. (2008) in Drosophila (Drosophila melanogaster). That study shows that a diet of $1: 2$ protein to carbohydrate maximizes reproductive rate but that a diet of $1: 16$ maximizes longevity (Lee et al. 2008). When given a mixed diet, female flies did not consume foods to reach either IT but rather traded off traits to maximize lifetime reproductive output, eating a diet of $1: 4$ protein to carbohydrate (Lee et al. 2008). Interestingly, dietary mediation of life-history tradeoffs may apply to many organisms, with similar results recently observed in mice (Solon-Biet et al. 2015).

Lefcheck et al. (2013) conclude that there is mixed evidence for physiological benefits of diet mixing and the balanced diet hypothesis. Our findings imply that there is considerable within-population variation in nutritional requirements and that a mixed diet allows an individual to reach its IT (maximizing fitness). Thus, in many species, it is possible that no one "ideal food" is able to perfectly meet the requirements of all individuals in a population. Accordingly, if some "optimal food" with a food rail passing directly through the species-mean IT were in the environment, high within-population variation in traits that correlate with nutritional requirements may prevent the species from specializing (fig. 3D). Importantly, the availability of multiple foods may itself promote within-population variation in nutritionally correlated traits, and where those traits have a genetic basis, the nutritional environment may facilitate the maintenance of genetic variation (fig. $3 D$ ).

In contrast to generalism, we predict that specialism is selected for in a highly constrained nutritional environment, for example, if confined to the environment in figure $3 B$, preference for food A would be selected against and preference for food B selected for. Given that variance in fitness on this single food would be high, over time (and subject to genetic constraints), the mean population IT may shift toward food B as the species adapts to maximize fitness on that food (Raubenheimer et al. 2012). Experimentally, physiological adaptations to dietary constraints have been observed within the time frame of a few generations (Warbrick-Smith et al. 2006). The hypothesis that dietary generalist strategies evolve in a complex nutritional environment accords with evidence from experimental evolution of generalism in other traits (Kassen 2002). Kassen (2002) reviewed such studies, finding that as a general rule (where genetic variance allows), generalism evolves in heterogeneous environments, whereas specialism evolves when constrained to a homogeneous environment.

Studying the performance of individuals with heterogeneous traits (correlated with the IT) on different diets may inform the relationship between the nutritional environment, within-population variance, and, ultimately, selection. These studies represent a complex challenge, as estimation of the IT is usually at the species level and involves maintaining individuals on the same diet throughout their life (Simpson and Raubenheimer 2012). Further, an individual's performance for some traits (e.g., longevity) cannot be independently measured on multiple diets. One solution may be to study individuals with phenotypes known to affect nutrient requirements. Further, where those phenotypes associate with specific loci, there is a unique opportunity to explore the effects of the nutritional environment on genetic diversity. In Drosophila, examples may include "rover" and "sitter" foraging phenotypes (Osborne et al. 1997) or individuals with mutations at Drosophila insulin-like peptide-encoding genes (Kannan and Fridell 2013). While experimental approaches are valuable, studying the nutritional choices of individuals in a field setting is also necessary. To date, few studies accurately quantify the nutritional decisions of individuals in the wild (e.g., Johnson et al. 2013). With wider application to wild populations, nutritional geometry may provide insight into the dietary constraints that animals face and the real-world costs (e.g., foraging time) associated with diet mixing.

Increasingly, evidence demonstrates that social interactions impact the nutrient acquisition of individuals, potentially affecting variance in nutritional states and fitness in social groups or populations (Lihoreau et al. 2014; Senior et al. 2015a). Using geometric agent-based models, Lihoreau et al. (2014) demonstrate how an optimal level of group cohesion can increase the efficiency of foraging decisions and decrease the costs of eating a balanced diet in a complex nutritional environment. However, high within-group variance in nutrient requirements might alter the cost-benefit tradeoff of social foraging decisions. In future, such geometric models might incorporate variance in ITs (Lihoreau et al. 2015).

Diet mixing can reduce between-individual variance in fitness, presumably because there is within-population variation in nutritional requirements and individuals selectively consume foods to meet their own needs. Where variance in nutrient requirements is associated with specific traits and/ or genotypes, a heterogeneous nutritional environment may contribute to within-population variation. Further, withinpopulation variation itself may help to explain the preva- 
lence of generalist dietary strategies. Nutritional geometry car. be a powerful conceptual framework for researchers to use in untangling the effects of diet on fitness in theoretical, experimental, and observational contexts.

\section{Acknowledgments}

We would like to thank S. Coogan, C. Grueber, R. O'Dea, S. Solon-Biet, two anonymous reviewers, and the editorial team at The American Naturalist for comments on the manuscript. S.J.S. was supported by an Australian Research Council Laureate Fellowship. S.N. was funded by a Rutherford Discovery Fellowship (New Zealand) and a Future Fellowship (Australia). M.L. is supported by grants from IDEXUniversity of Toulouse and the Fyssen Foundation. D.R. is an affiliate of the New Zealand Institute for Advanced Study.
Higgins, J. P. T., and S. G. Thompson. 2002. Quantifying heterogeneity in a meta-analysis. Statistics in Medicine 21:1539-1558.

Honěk, A. 1993. Intraspecific variation in body size and fecundity in insects: a general relationship. Oikos 66:483-492.

$\rightarrow$ Houston, A. I., A. D. Higginson, and J. M. McNamara. 2011. Optimal foraging for multiple nutrients in an unpredictable environment. Ecology Letters 14:1101-1107.

$\rightarrow$ Huchard, E., A. Charmantier, S. English, A. Bateman, J. F. Nielsen, and T. Clutton-Brock. 2014. Additive genetic variance and developmental plasticity in growth trajectories in a wild cooperative mammal. Journal of Evolutionary Biology 27:1893-1904.

Jennions, M. D., C. J. Lortie, M. S. Rosenberg, and H. R. Rothstein. 2012. Publication and related biases. Pages 207-236 in J. Koricheva, J. Gurevitch, and K. Mengersen, eds. The handbook of meta-analysis in ecology and evolution. Princeton University Press, Princeton, NJ.

Jensen, K., D. Mayntz, S. Toft, F. J. Clissold, J. Hunt, D. Raubenheimer, and S. J. Simpson. 2012. Optimal foraging for specific nutrients in predatory beetles. Proceedings of the Royal Society B: Biological Sciences 279:2212-2218.

$\rightarrow$ Johnson, C. A., D. Raubenheimer, J. M. Rothman, D. Clarke, and L. Swedell. 2013. 30 days in the life: daily nutrient balancing in a wild chacma baboon. PLoS ONE 8:e70383.

\section{Literature Cited}

Boyle, M. A., A. L. Samaha, A. M. Rodewald, and A. N. Hoffmann. 2013 Evaluation of the reliability and validity of GraphClick as a data extraction program. Computers in Human Behavior 29:1023-1027.

$\rightarrow$ Cam, E., W. A. Link, E. G. Cooch, J.-Y. Monnat, and E. Danchin 2002. Individual covariation in life-history traits: seeing the trees despite the forest. American Naturalist 159:96-105.

$\rightarrow$ Day, J. E. L., I. Kyriazakis, and P. J. Rogers. 1998. Food choice and in take: towards a unifying framework of learning and feeding motivation. Nutritional Research Reviews 11:25-43.

$\rightarrow$ DeMott, W. R. 1998. Utilization of a cyanobacterium and a phosphorusdeficient green alga as complementary resources by daphnids. Ecol ogy 79:2463-2481.

$\rightarrow$ Dennis, R. L. H., L. Dapporto, S. Fattorini, and L. M. Cook. 2011. The generalism-specialism debate: the role of generalists in the life anc $\rightarrow$ death of species. Biological Journal of the Linnean Society 104:725737.

$\rightarrow$ Dussutour, A., and S. J. Simpson. 2009. Communal nutrition in ants. Current Biology 19:740-744

$\rightarrow$ Egger, M., G. D. Smith, M. Schneider, and C. Minder. 1997. Bias in meta $\rightarrow$ analysis detected by a simple, graphical test. BMJ 315:629-634.

Gleser, L. J., and I. Olkin. 2009. Stochastically dependent effect sizes. Pages 357-376 in H. Cooper, L. V. Hedges, and J. C. Valentine, eds The handbook of research synthesis and meta-analysis. Russell Sage Foundation, New York.

$\rightarrow$ Grueber, C. E., S. Nakagawa, R. J. Laws, and I. G. Jamieson. 2011. Multimodel inference in ecology and evolution: challenges and solutions. Journal of Evolutionary Biology 24:699-711.

$\rightarrow$ Hadfield, J. D. 2010. MCMC methods for multi-response generalized linear mixed models: the MCMCglmm R package. Journal of Statistical Software 33:1-22.

$\rightarrow$ Hadfield, J. D., and S. Nakagawa. 2010. General quantitative ge netic methods for comparative biology: phylogenies, taxonomies and multi-trait models for continuous and categorical characters. Journal of Evolutionary Biology 23:494-508.

Hallgrimsson, B., and K. H. Brian. 2005. Variation and variability: central concepts in biology. Pages 1-7 in B. Hallgrimsson and K. H. Brian, eds. Variation: a central concept in biology. Elsevier Academic Burlington, MA.
Kannan, K., and Y.-W. Fridell. 2013. Functional implications of Drosophila insulin-like peptides in metabolism, aging and dietary restriction. Frontiers in Physiology 4:288. and the maintenance of diversity. Journal of Evolutionary Biology 15:173-190.

Lajeunesse, M. J. 2011. On the meta-analysis of response ratios for studies with correlated and multi-group designs. Ecology 92:2049-2055.

Lajeunesse, M. J., M. S. Rosenberg, and M. D. Jennions. 2013. Phylogenetic nonindependence and meta-analysis. Pages 284-299 in J. Koricheva, J. Gurevitch, and K. Mengersen, eds. Handbook of meta-analysis in ecology and evolution. Princeton University Press, Princeton, NJ.

Lee, K. P., S. J. Simpson, F. J. Clissold, R. Brooks, J. W. O. Ballard, P. W. Taylor, N. Soran, and D. Raubenheimer. 2008. Lifespan and reproduction in Drosophila: new insights from nutritional geometry. Proceedings of the National Academy of Sciences of the USA 105: 2498-2503.

Lefcheck, J. S., M. A. Whalen, T. M. Davenport, J. P. Stone, and J. E. Duffy. 2013. Physiological effects of diet mixing on consumer fitness: a meta-analysis. Ecology 94:565-572.

Liberati, A., D. G. Altman, J. Tetzlaff, C. Mulrow, P. C. Gotzsche, J. P. A. Ioannidis, M. Clarke, P. J. Devereaux, J. Kleijnen, and D. Moher. 2009. The PRISMA statement for reporting systematic reviews and meta-analyses of studies that evaluate health care interventions: explanation and elaboration. PLoS Medicine 6:e1000100.

$\rightarrow$ Lihoreau, M., J. Buhl, M. A. Charleston, G. A. Sword, D. Raubenheimer, and S. J. Simpson. 2014. Modelling nutrition across organizational level: from individuals to superorganisms. Journal of Insect Physiology 69:2-11.

2015. Nutritional ecology beyond the individual: a conceptual framework for integrating nutrition and social interactions. Ecology Letters 18:273-286.

Lim, J. N., A. M. Senior, and S. Nakagawa. 2014. Heterogeneity in individual quality and reproductive tradeoffs within species. Evolution 68:2306-2318.

Maklakov, A. A., S. J. Simpson, F. Zajitschek, M. D. Hall, J. Dessmann, F. Clissold, D. Raubenheimer, R. Bonduriansky, and R. C. Brooks. 2008.
Kassen, R. 2002. The experimental evolution of specialists, generalists, 
Sex-specific fitness effects of nutrient intake on reproduction and lifespan. Current Biology 18:1062-1066.

$\rightarrow$ Mathot, K. J., and N. J. Dingemanse. 2015. Energetics and behavior: unrequited needs and new directions. Trends in Ecology and Evolution 30:199-206.

$\rightarrow$ Nakagawa, S., and R. Poulin. 2012. Meta-analytic insights into evolution ary ecology: an introduction and synthesis. Evolutionary Ecology 26:1085-1099.

$\rightarrow$ Nakagawa, S., R. Poulin, K. Mengersen, K. Reinhold, L. Engqvist, M Lagisz, and A. M. Senior. 2015. Meta-analysis of variation: ecological and evolutionary applications and beyond. Methods in Ecology and Evolution 6:143-152.

$\rightarrow$ Nakagawa, S., and E. S. A. Santos. 2012. Methodological issues and advances in biological meta-analysis. Evolutionary Ecology 26:12531274

$\rightarrow$ Orr, H. A. 2009. Fitness and its role in evolutionary genetics. Nature Reviews Genetics 10:531-539.

$\rightarrow$ Osborne, K. A., A. Robichon, E. Burgess, S. Butland, R. A. Shaw, A. Coulthard, H. S. Pereira, R. J. Greenspan, and M. B. Sokolowski. 1997. Natural behavior polymorphism due to a cGMP-dependen $\rightarrow$ protein kinase of Drosophila. Science 277:834-836.

$\rightarrow$ Raubenheimer, D., D. Mayntz, S. J. Simpson, and S. Toft. 2007. Nutrientspecific compensation following diapause in a predator: implication for intraguild predation. Ecology 88:2598-2608

$\rightarrow$ Raubenheimer, D., and S. J. Simpson. 1993. The geometry of com pensatory feeding in the locust. Animal Behaviour 45:953-964.

$\rightarrow$ Raubenheimer, D., S. J. Simpson, and A. H. Tait. 2012. Match and mis match: conservation physiology, nutritional ecology and the timescales of biological adaptation. Philosophical Transactions of the Royal Society B: Biological Sciences 367:1628-1646.

R Development Core Team. 2014. R: a language and environment fo statistical computing. Version 3.1.1 http://www.r-project.org.

$\rightarrow$ Schielzeth, H. 2010. Simple means to improve the interpretability of regression coefficients. Methods in Ecology and Evolution 1:103-113.

$\rightarrow$ Senior, A. M., M. A. Charleston, M. Lihoreau, J. Buhl, D. Raubenheimer and S. J. Simpson. 2015a. Evolving nutritional strategies in the presence of competition: a geometric agent-based model. PLoS Computational Biology 11:e1004111.
Senior, A. M., S. Nakagawa, M. Lihoreau, S. J. Simpson, and D. Raubenheimer. 2015b. Data from: An overlooked consequence of dietary mixing: a varied diet reduces interindividual variance in fitness. American Naturalist, Dryad Digital Repository, http://dx.doi .org/10.5061/dryad.9hc76.

Simpson, S. J., F. Clissold, M. Lihoreau, F. Ponton, S. M. Wilder, and D. Raubenheimer. 2015. Recent advances in the integrative nutrition of arthropods. Annual Review of Entomology 60:293-311.

Simpson, S. J., and D. Raubenheimer. 2012. The nature of nutrition: a unifying framework from animal adaptations to human obesity. Princeton University Press, Oxford.

$\rightarrow$ Simpson, S. J., R. M. Sibly, K. P. Lee, S. T. Behmer, and D. Raubenheimer. 2004. Optimal foraging when regulating intake of multiple nutrients. Animal Behaviour 68:1299-1311.

$\rightarrow$ Solon-Biet, S. M., K. A. Walters, U. Simanainen, A. C. McMahon, K. Ruohonen, J. W. O. Ballard, D. Raubenheimer, D. J. Handelsman, D. G. Le Couteura, and S. J. Simpson. 2015. Macronutrient balance, reproductive function and lifespan in aging mice. Proceedings of the National Academy of Sciences of the USA 112:3481-3486.

Spiegelhalter, D. J., N. G. Best, B. P. Carlin, and A. Van Der Linde. 2002. Bayesian measures of model complexity and fit. Journal of the Royal Statistical Society B: Statistical Methodology 64:583-639.

Van Valen, L. 1965. Morphological variation and width of ecological niche. American Naturalist 99:377-390.

$\rightarrow$ Viechtbauer, W. 2010. Conducting meta-analyses in R with the metafor package. Journal of Statistical Software 36:1-48.

$\rightarrow$ Violle, C., B. J. Enquist, B. J. McGill, L. Jiang, C. H. Albert, C. Hulshof, V. Jung, and J. Messier. 2012. The return of the variance: intraspecific variability in community ecology. Trends in Ecology and Evolution 27:244-252.

Warbrick-Smith, J., S. T. Behmer, K. P. Lee, D. Raubenheimer, and S. J. Simpson. 2006. Evolving resistance to obesity in an insect. Proceedings of the National Academy of Sciences of the USA 103:1404514049. 